Reprod. Nutr. Dévelop., 1986, 26 (1 B), 343-344.

\title{
Relation entre les teneurs en acides aminés libres du sang et la production de lait chez la chèvre en $4^{e}$ semaine de lactation
}

\author{
D. SAUVANT, J.-C. ROBERT $\left({ }^{*}\right)$
}

Avec la collaboration technique de Françoise TERNOIS, Madeleine DURON (*), J. HERVIEU

Station de Nutrition et Alimentation I.N.R.A. Institut National Agronomique Paris-Grignon 16, rue Claude Bernard, 75231 Paris Cédex 05 (*) A.E.C. Rhône-Poulenc 03600 Commentry.

Summary. Blood free amino acids (BFAA) were measured on goats during the 4th week of lactation. At that time, lipomobilization parameters were not related to milk yield which was correlated negatively with plasma glucose and varied with the BFAA content. The probable amino acid extraction rate of the udder largely accounted for the variations in the relationship between milk yield and BFAA. Histidine appeared to be the main limiting aminocacid.

La teneur du sang en acides aminés libres ( $A A L)$ est mesurée pour apprécier l'état de nutrition azotée des animaux domestiques. Une telle mesure individuelle a été notamment appliquée chez la chèvre au début de la lactation par Champredon et Pion (1979) et Sauvant et Robert (1983). Les résultats décrits correspondent à des mesures effectuées sur des chèvres en $4^{\mathrm{e}}$ semaine de lactation.

Matériel et méthodes. Vingt huit chèvres Alpines ont reçu, pendant la semaine considérée, une ration constituée en moyenne, sur la base de la matière sèche, $20 \%$ de foin de luzerne, de $38 \%$ de maïs fourrage ensilé et de $42 \%$ d'un aliment concentré, de taux azoté égal à $21 \%$ de la MS. Le rapport PDIEPDIN/UFL de la ration était de +3 .

Les prélèvements de sang ont été effectués au milieu de la $4^{\mathrm{e}}$ semaine, après la traite du matin, avant le premier repas de foin. On a dosé les teneurs plasmatiques en glucose, AGNE, B-OH-butyrate, urée et les teneurs en acides aminés libres du sang.

Résultats et discussion. Les paramètres statistiques des principaux caractères considérés sont rapportés dans le tableau 1. Les variations individuelles du bilan énergétique calculé sont significativement corrélées aux caractéristiques térnoins de la lipomobilisation, les $\operatorname{AGNE}(-0,56)$, le B-OH-butyrate $(-0,40)$ et la proportion d'acide oléique dans la matière grasse du lait $(-0,74)$. Les variations de la production de lait (brut ou corrigé) sont positivement corrélées à la quantité de M.S. ingérée $(0,69)$ mais ne sont pas liées aux bilans énergétique et PDI ainsi qu'aux paramètres de la lipomobilisation. D'autre part, la glycémie est significativement corrélée avec la production de lait $(-0,52)$ et l'urémie $(0,73)$.

La teneur en valine du sang est négativement liée aux témoins de la lipomobilisation, confirmant la relation observée à un stade plus précoce de la lactation (Sauvant et Robert, 1983). Des relations plus étroites associent les teneurs en acides aminés libres avec la production de lait et la glycémie. Le niveau de production de lait présente un large éventail, régulièrement réparti, de valeurs de corrélation avec les teneurs en AAL du sang (tabl. 1) : de + 0,73 avec la glycine à 0,61 avec l'histidine qui apparaît donc comme l'acide aminé le plus limitant dans les conditions expérimentales décrites. Ce rôle de I'histidine n'a pas été observé au même stade physiologique par Laurent et al. (non publié), peut-être en raison 
de la différence d'heure de prélèvement du sang qui est effectué par ces auteurs en milieu de journée sur des animaux ayant déjà effectué un repas. Les autres acides aminés essentiels, mis à part la thréonine, sont corrélés négativement à la production laitière (tabl. 1). Champredon et Pion (1979) ont notamment cité I'histidine et la phénylalanine, mais aussi la thréonine, comme acide aminé limitant probable après la méthionine. D'autres caractéristiques telles que la quantité de PDI ingérée, le bilan PDI calculé, l'urémie ou la sécrétion de matière protéique par la mamelle sont moins corrélés aux AAL que la production de lait et la glycémie.

TABL. 1. - Paramètres statistiques des principales caractéristiques et leur corrélation $(R)$ avec la quantité de lait produit

\begin{tabular}{|c|c|c|c|c|c|}
\hline & Moyenne & Écart-type & Minimum & Maximum & $\mathrm{R}$ \\
\hline Matière sèche ingérée $(\mathrm{kg} / \mathrm{j})$ & 2,18 & 0,32 & 1,29 & 2,52 & $0,69 * *$ \\
\hline Bilan énergétique calculé (Mcal/j) & $-0,65$ & 0,36 & $-1,64$ & $-0,10$ & $-0,32^{*(L)}$ \\
\hline Bilan PDI calculé $(\mathrm{g} / \mathrm{j})$ & 39,4 & 24,0 & 0,8 & 91,7 & $-0,36^{*}$ \\
\hline Quantité de lait $(\mathrm{kg} / \mathrm{j})$ & 3,21 & 0,85 & 0,90 & 4,56 & 1,00 \\
\hline \multicolumn{6}{|l|}{ Acides aminés libres (mg/100 g) } \\
\hline ASN & 0,26 & 0,07 & 0,10 & 0,42 & 0,25 \\
\hline THR & 0,92 & 0,25 & 0,42 & 1,51 & 0,26 \\
\hline SER & 1,59 & 0,55 & 0,50 & 2,67 & $0,60^{* *}$ \\
\hline ASP & 0,92 & 0,35 & 0,27 & 1,73 & $0,39^{*}$ \\
\hline GLU & 2,13 & 0,51 & 1,13 & 3,26 & $-0,15$ \\
\hline PRO & 1,44 & 0,32 & 0,64 & 1,88 & 0,27 \\
\hline GLY & 6,46 & 1,52 & 2,21 & 8,65 & $0,73^{* *}$ \\
\hline ALA & 1,41 & 0,34 & 0,72 & 1,90 & $-0,04$ \\
\hline $\mathrm{CIT}$ & 2,08 & 0,69 & 0,72 & 3,78 & 0,22 \\
\hline VAL & 1,39 & 0,43 & 0,47 & 2,35 & $-0,29$ \\
\hline MET & 0,46 & 0,15 & 0,21 & 0,76 & 0,03 \\
\hline ILE & 1,02 & 0,27 & 0,25 & 1,35 & 0,18 \\
\hline LEU & 1,30 & 0,31 & 0,69 & 1,84 & 0,03 \\
\hline TYR & 0,76 & 0,22 & 0,32 & 1,27 & $-0,11$ \\
\hline PHE & 0,64 & 0,11 & 0,46 & 0,91 & $-0,33^{*(L)}$ \\
\hline ORN & 0,53 & 0,18 & 0,10 & 0,79 & 0,21 \\
\hline LYS & 1,85 & 0,42 & 1,15 & 2,72 & 0,04 \\
\hline HIS & 0,52 & 0,19 & 0,29 & 1,06 & $-0,61^{* *}$ \\
\hline ARG & 1,78 & 0,44 & 1,01 & 2,62 & $-0,21$ \\
\hline
\end{tabular}

(1) Limite de signification à $P<0,05^{*}, P<0,01^{* *}$

Il semble que le taux d'extraction des AAL par la mamelle soit un paramètre déterminant de leur teneur sanguine après le jeûne nocturne. En effet, une relation significative $(-0,56)$ associe les valeurs des corrélations (production-teneur en $\mathrm{AAL}$ ) et les mesures des taux d'extraction d'AAL par la mamelle de chèvre obtenus par Linzell (1968), taux globalement confirmés par différents travaux publiés sur ce sujet. La relation est encore plus précise lorsque l'histidine est exclue de cette relation $(-0,70)$ en raison de sa position significativement aberrante par rapport aux autres acides aminés. Cette position de l'histidine, dont la corrélation avec la production de lait est plus négative que ne le laisse supposer son taux probable d'extraction, pourrait confirmer son rôle de facteur limitant primaire de la production de lait dans les conditions expérimentales présentes.

Champredon C., Pion R., 1979. Intravenous infusion of amino-acids to lactating goats. Ann. Rech. vét., 10, 379-381.

Linzell J. L., 1968. The magnitude and mechanisms of the uptake of milk percursors by the mammary gland. Proc. Nutr. Soc., 21, 44-52.

Sauvant D., Robert J.-C., 1983. Etude chez la chèvre en début de lactation, des variations individuelles de la teneur en acides aminés libres du sang. Ne Symp. Int. Métabolisme et nutrition azotés. Ed. I.N.R.A., les Colloques de I'I.N.R.A. No 16. 